\title{
Abl expression, tumour grade, and apoptosis in chondrosarcoma
}

\author{
M O’Donovan, J M Russell, J J O’Leary, J A Gillan, M P Lawler, E F Gaffney
}

\begin{abstract}
Aims-To determine whether Abl immunoreactivity correlates with grade and cell kinetics (apoptosis and mitosis) in chondrosarcoma.

Methods-Sections from 16 chondrosarcomas were stained immunohistochemically using a polyclonal antibody to the c-Abl/Bcr-Abl oncoprotein. Apoptotic indices and mitotic indices were assessed in all tumours. Sections from 24 paraffin wax blocks of human fetal rib (gestational ages, 15-42 weeks) were also stained to determine whether the Abl protein is synthesised consistently throughout endochondral ossification.

Results-Abl staining in immature fetal rib chondrocytes at all stages of development was predominantly nuclear, and $70 \%$ of cells showed moderate to strong staining. Abl immunoreactivity was minimal or absent in hypertrophic chondrocytes about to undergo apoptosis at the growth plate. There was strong Abl staining in grade 1 and grade 2 chondrosarcomas but staining was greatly reduced or absent in grade 3 chondrosarcomas. There was a very significant linear correlation between apoptotic index (mean, $0.68 \%$; range, $0-3.2 \%$ ) and mitotic index (mean, $0.23 \%$; range, $0-0.9 \%$ ), and both indices were significantly lower in grade 1 than in grade 2 and grade 3 chondrosarcomas.

Conclusions-These data suggest that abl gene expression is associated with differentiation and apoptosis inhibition in fetal and neoplastic chondrocytes. However, these putative effects cannot be ascribed solely to the Abl protein, because several additional factors contribute to the regulation of both differentiation and apoptosis.

(F Clin Pathol: Mol Pathol 1999;52:341-344)
\end{abstract}

Keywords: abl gene expression; chondrosarcoma; apoptosis

The abl oncogenes are cellular homologues of the v-abl oncogene of the Abelson murine leukaemia retrovirus. They encode nuclear and cytoplasmic protein tyrosine kinases that function in signal transduction, cell cycle dependent and DNA damage induced gene expression, and apoptosis inhibition. ${ }^{1-6}$ The biological effects of c-Abl vary in different cell types and may depend on an equilibrium between nuclear and cytoplasmic c-Abl. ${ }^{5}$ The bcr-abl fusion gene occurs in $90 \%$ of cases of chronic myeloid leukaemia, as a result of reciprocal translocation of c-abl (chromosome 9) to the breakpoint cluster region, bcr on chromosome $22 .{ }^{3}$ bcr-abl encodes the chimaeric protein $\mathrm{Bcr}-\mathrm{Abl}$, which has enhanced protein tyrosine kinase activity and suppresses apoptosis in the indolent phase of chronic myeloid leukaemia. ${ }^{3}$

Recently, it has been reported that c-Abl proteins, encoded by the ubiquitous $\mathrm{c}-\mathrm{abl}$ proto-oncogene, also inhibit apoptosis. ${ }^{78} \mathrm{We}$ have previously reported variable Abl immunoreactivity in a wide range of normal human fetal and adult tissues and tumours. ${ }^{9}$ Intense Abl immunoreactivity was seen in fetal and adult chondrocytes and in low grade chondrosarcomas. ${ }^{9}$

The purpose of our study was to determine whether Abl is: (1) synthesised consistently in sequential stages of fetal chondrocyte maturation, and (2) related to grade and tumour cell kinetics (apoptosis and mitosis) in chondrosarcoma.

\section{Materials and methods}

TISSUE SAMPLES

Twenty four formalin fixed paraffin wax embedded blocks of normal fetal rib taken at postmortem were obtained from the files of the department of histopathology, Rotunda Hospital, Dublin. Specimens were selected to encompass gestational ages from 15 to 42 weeks. The material was well fixed, showing minimal autolysis.

Chondrosarcomas were retrieved from the files of the departments of histopathology, St James's Hospital and Mater Misericordiae Hospital, Dublin. Of 20 cases diagnosed from 1989 to 1997,16 (femur (four), rib (three), ilium (two), humerus (two), scapula (two), fibula (one), sites not recorded (two)) had sufficient well preserved formalin fixed tissue for our study. Tumours were graded using standard criteria recently summarised by Bjornsson and colleagues ${ }^{10}$ as follows: six grade 1 , five grade 2, and five grade 3 (including one dedifferentiated chondrosarcoma). From each case, one paraffin wax block was selected for $\mathrm{Abl}$ immunostaining and assessment of apoptosis and mitosis.

\section{IMMUNOHISTOCHEMISTRY}

Paraffin wax sections ( $4 \mu \mathrm{m}$ thick) were cut and mounted on 3-aminopropyltriethoxysilane coated slides, oven dried, dewaxed in xylene, and rehydrated in alcohol. The immunostaining protocol has been described previously. ${ }^{9}$ Briefly, sections were incubated with a polyclonal sheep anti-c-Abl/Bcr-Abl oncoprotein antibody (Serotec, Oxford, UK; 1/200 dilution), for one hour at room temperature, then incubated with biotinylated rabbit anti-sheep antibody (Serotec; $1 / 400$ ), followed by peroxidase conjugated
Accepted for publication 15 July 1999 
streptavidin (1/400; Dako, Glostrup, Denmark). The reaction product was visualised with 3,3'-diaminobenzidine and a haematoxylin counterstain. Appropriate positive and negative controls were used, as described previously. ${ }^{9} \mathrm{Abl}$ immunoreactivity was assessed using a three tiered scale: + (focal or diffuse weak staining), ++ (moderately intense staining), and +++ (intense staining).

\section{APOPTOSIS AND MITOSIS ASSESSMENT}

The extent of apoptosis was assessed in all 16 tumours by counting tumour cells in 10 high power fields using the $\times 40$ objective with a 100 square graticule. ${ }^{11}$ We decided to use haematoxylin and eosin stained sections, and not in situ end labelling of fragmented apoptotic cell DNA, because apoptosis and mitosis could be assessed in identical microscopic fields. The apoptotic index assigned to each sample represents the total number of apoptotic tumour cells and apoptotic bodies expressed as a percentage of the total number of tumour cells in 10 fields. ${ }^{11}$ The mitotic index was determined using the same procedure.

From raw data, the relation between the apoptotic index and the mitotic index was analysed using parametric (Pearson) linear correlations (Instat, Graphpad Software, San Diego, California, USA). From contingency tables, Fisher's exact test was used to compare grade 1 chondrosarcomas and grades $2 / 3$ chondrosarcomas (rows) with apoptotic index $=0$ and apoptotic index $>0$ (columns), and also with mitotic index $=0$ (rows) and mitotic index $>0$ (columns).

\section{Results}

FETAL CARTILAGE

Abl staining was predominantly nuclear, although cytoplasmic or cell membrane staining was found occasionally. Most epiphyseal reserve chondrocytes and proliferating immature chondrocytes in fetuses of all ages showed moderate to strong $\mathrm{Abl}$ immunoreactivity (fig 1A). In contrast, hypertrophic chondrocytes about to undergo apoptosis during enchondral ossification showed minimal or no Abl staining (fig 1B). There was intense Abl staining of adjacent proliferating osteoblasts and invading metaphyseal blood vessels, as seen previously. ${ }^{9}$

ABL EXPRESSION, GRADE, APOPTOSIS, AND MITOSIS IN CHONDROSARCOMA

In all six grade 1 chondrosarcomas, more than $70 \%$ of tumour cells showed intense (3+) nuclear Abl staining (table 1). Grade 2 chondrosarcomas showed variably intense nuclear Abl staining: $3+$ in one tumour and $2+$ in the other four samples (fig 1C). In grade 3 chondrosarcomas, Abl staining was negative (fig 1D) or, in one case, very weak $(1+)$. The dedifferentiated chondrosarcoma showed intense (3+) $\mathrm{Abl}$ immunoreactivity in the low grade component, but no staining in the high grade component (fig 1E).

Mean apoptotic indices and mitotic indices (and ranges) for the 16 chondrosarcomas (table 1 ) were $0.68 \%(0-3.2 \%)$ and $0.23 \%$ $(0-0.9 \%)$, respectively. In all tumours except one the number of mitoses was less than or equivalent to the number of apoptotic tumour cells. There was a very significant linear correlation between the apoptotic and mitotic indices $(\mathrm{p}<0.0090 ; r=0.6294))$.
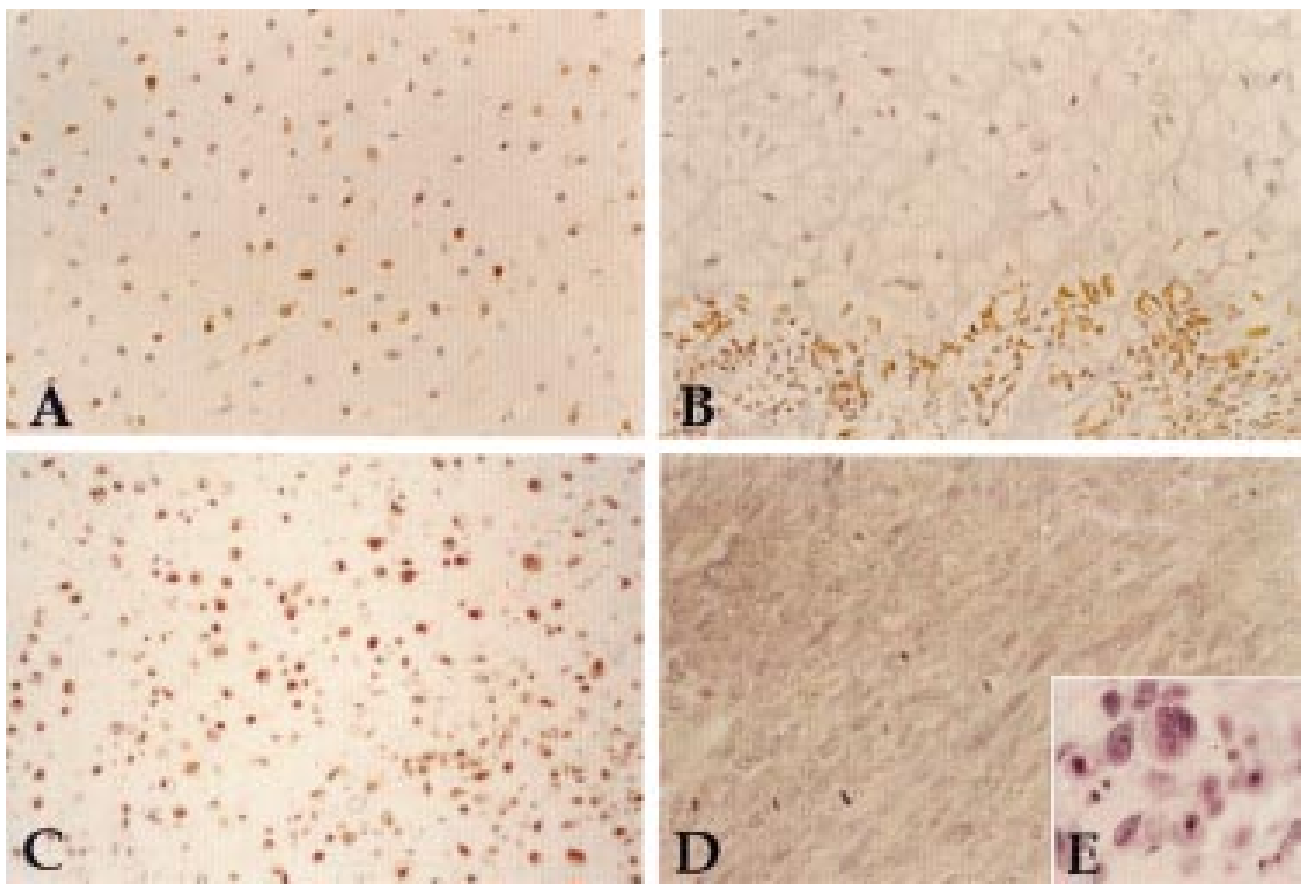

Figure 1 (A) Immature chondrocytes in the developing human fetal rib show nuclear, cell membrane, or cytoplasmic immunoreactivity for Abl. (B) Hypertrophic chondrocytes show minimal or no Abl staining in sites of enchondral ossification, whereas osteoblasts and proliferating blood vessels (below) are stained intensely. (C) Variably intense Abl immunoreactivity in grade 2 chondrosarcoma. (D) Abl immunoreactivity is absent in grade 3 chondrosarcoma. (E) Numerous apoptotic tumour cells in the high grade component of dedifferentiated chondrosarcoma. 
Table 1 Apoptotic indices (AI), mitotic indices (MI), and Abl immunoreactivity in 16 chondrosarcomas

\begin{tabular}{lllll}
\hline $\begin{array}{l}\text { Case } \\
\text { no. }\end{array}$ & Grade & $\begin{array}{l}\text { Abl } \\
\text { immunoreactivity }\end{array}$ & AI (\%) & MI (\%) \\
\hline 1 & G1 & $3+$ & 0 & 0 \\
2 & G1 & $3+$ & 0 & 0 \\
3 & G1 & $3+$ & 0 & 0 \\
4 & G1 & $3+$ & 0 & 0 \\
5 & G1 & $3+$ & 0 & 0 \\
6 & G1 & $3+$ & 0 & 0 \\
7 & G2 & $3+$ & 0 & 0 \\
8 & G2 & $2+$ & 0 & 0 \\
9 & G2 & $2+$ & 0 & 0 \\
10 & G2 & $2+$ & 0.6 & 0.2 \\
11 & G2 & $2+$ & 1.9 & 0.6 \\
12 & G3 & $1+$ & 0.8 & 0.3 \\
13 & G3 & Negative & 0.3 & 0.9 \\
14 & G3 & Negative & 2.8 & 0.8 \\
15 & G3 & Negative & 3.2 & 0.3 \\
16 & G3 & Negative & 1.2 & 0.5 \\
\hline
\end{tabular}

The extent of both apoptosis and mitotic activity correlated with chondrosarcoma grade (fig 1E; table 1). There was no apoptosis or mitotic activity in grade 1 chondrosarcomas. Grade 2 chondrosarcomas (apoptotic index, $0.5 \%$; range, $0-1.9 \%$; mitotic index, $0.16 \%$; range, $0-0.6 \%$ ) and grade 3 chondrosarcomas (apoptotic index, 1.7\%; range, 0.3-3.2\%; mitotic index, $0.6 \%$; range: $0.3-0.9 \%$ ) combined had significantly higher apoptotic indices $(\mathrm{p}<0.0114)$ and mitotic indices $(\mathrm{p}<0.0114)$ than grade 1 tumours.

\section{Discussion}

It has been confirmed during the past five years that terminally differentiated hypertrophic chondrocytes at the chondro-osseous junction die by undergoing apoptosis. ${ }^{12-16}$ Apoptosis occurs before, or simultaneously with, the resorption of chondrocytes and the invasion by capillaries and proliferating osteoblasts at the metaphysis. ${ }^{16}$ Endochondral ossification is regulated by bone morphogenetic proteins, ${ }^{17}$ but the stimulus that induces apoptosis in terminally differentiated chondrocytes is unknown. ${ }^{16}{ }^{18}$ Possibilities include downregulation of the bcl-2 gene, which occurs in the late hypertrophic stage, ${ }^{14}$ downregulation of C-Jun and Jun D, which might permit chondrocyte maturation and apoptosis with terminal differentiation, ${ }^{19}$ and downregulation of c-abl.

Our results suggest strongly that the c-Abl protein plays a key role in chondrocyte differentiation and apoptosis inhibition in fetal cartilage. Immature human fetal rib chondrocytes at all developmental stages examined (15-42 weeks) showed moderate to strong $\mathrm{c}-\mathrm{Abl}$ staining without apoptosis. In contrast, hypertrophic chondrocytes about to undergo apoptosis during endochondral ossification showed minimal or no Abl immunoreactivity. Although there are many potential initiators and inducers of apoptosis in chondrocytes, ${ }^{20}$ loss of Abl staining in hypertrophic chondrocytes is likely to be important, in that such cells no longer need to avoid undergoing apoptosis, having become fully (terminally) differentiated. Furthermore, the distribution of Abl staining is most consistent with the developing chondrocytes' progressive requirements for maturation, terminal differentiation, and apoptosis.
There was intense $\mathrm{Abl}$ immunoreactivity in grade 1 and grade 2 chondrosarcomas, but very weak or no Abl staining in grade 3 chondrosarcomas. Therefore, Abl immunoreactivity in these malignancies correlates with differentiation, as in developing fetal chondrocytes. Abl production in grade 1 chondrosarcoma was associated with the absence of apoptosis and mitosis. The anti-apoptotic effects of Abl are well described, ${ }^{1347}$ and $\mathrm{Abl}$ is also reported to exert an antiproliferative effect on fibroblasts. ${ }^{2} 6$ In contrast, absent or minimal Abl immunoreactivity in grade 3 chondrosarcoma was associated with conspicuous apoptosis, suggesting that a large reduction or absence of the Abl protein increases the susceptibility of tumour cells to apoptosis in grade 3 chondrosarcoma. Using TaqMan reverse transcription polymerase chain reaction analysis, ${ }^{21}$ and paraffin wax embedded tumour tissue, we have demonstrated the expression of c-abl mRNA in three grade 1 chondrosarcomas (data not shown), but because of difficulties with RNA extraction we were unsuccessful with other specimens. Therefore, we cannot confirm that c-abl mRNA is downregulated in higher grade tumours. Our results suggest an apoptosis inhibiting function for c-Abl in chondrosarcoma, but it is likely that the conspicuous apoptosis and greater mitotic activity of high grade chondrosarcomas involves the participation of additional gene products and other factors associated with increased genetic instability. $^{22}$

There was a very significant linear correlation between apoptotic and mitotic indices in chondrosarcoma. A similar relation has been reported in malignant lymphoma and in various carcinomas, ${ }^{23-26}$ supporting the concept that cellular proliferative and apoptotic pathways are coupled..$^{27}$ In chondrosarcoma, and in cancer in general, apoptotic indices are typically higher than or similar to mitotic indices ${ }^{11}$ because apoptotic cells persist longer in tissues than mitoses, although there is a disproportionately greater increase in mitoses over time to facilitate tumour growth. ${ }^{22}$ It is well known that grade 1 chondrosarcomas have no mitoses and low proliferative indices. ${ }^{10}{ }^{28}$ We now report that grade 1 tumours have no apoptosis which, taken together with the absence of mitoses and low proliferative indices, is compatible with slow growth and indolent biological behaviour. In grade 2 and 3 chondrosarcomas, both apoptotic and mitotic indices were relatively low, but significantly higher than in grade 1 tumours, and the difference in cell kinetics reflects the potential for the more aggressive biological behaviour associated with high grade tumours. ${ }^{10}$

In summary, our results suggest that $\mathrm{Abl}$ immunoreactivity correlates with differentiation in both fetal chondrocytes and chondrosarcomas. Absent Abl staining correlates with terminal differentiation and apoptosis in developing cartilage and with high chondrosarcoma grade. However, these putative effects cannot be ascribed solely to Abl, because several additional factors contribute to the regulation of both differentiation and apoptosis. 
This work was supported by the Health Research Board (Ireland). We wish to thank Professor P Dervan (Mater Misericordiae Hospital, Dublin) for providing slides and blocks from 11 chondrosarcomas. Presented in part at the 175th meeting of the Pathological Society of Great Britain and Ireland, Sheffield, 2nd July, 1997.

1 Wang JYJ. Abl tyrosine kinase in signal transduction and cell-cycle regulation. Curr Opin Genet Dev 1993;3:35-43.

2 Sawyers CL, McLaughlin J, Goga A, et al. The nuclear tyrosine kinase c-Abl negatively regulates cell growth. Cell 1994;77:121-31.

3 Mc Gahon AJ, Cotter TG, Green DR. The abl oncogene family and apoptosis. Cell Death and Differentiation 1994;1: $77-83$

4 Bedi A, Zehnbauer BA, Barber JP, et al. Inhibition of apoptosis by BCR-ABL in chronic myeloid leukaemia. Blood 1994;83:2038-44.

5 Taagepera S, McDonald D, Loeb JE, et al. Nuclearcytoplasmic shuttling of C-ABL tyrosine kinase. Proc Nat Acad Sci USA 1998;95:7457-62.

6 Wen S-T, Jackson PK, Van Etten RA. The cytostatic function of c-Abl is controlled by multiple nuclear localization signals and requires the $\mathrm{p} 53$ and $\mathrm{Rb}$ tumor suppressor tion signals and requires the p 53 and Rb tu
gene products. $E M B O \mathcal{1} 1996 ; 15: 1583-95$.

7 Daniel R, Wong PMC, Chung S-W. Isoform-specific functions of c-abl: type I is necessary for differentiation, functions of c-abl: type I is necessary for differentiation,
and type II is inhibitory to apoptosis. Cell Growth Differ and type II is 1 in

8 Chung S-W, Daniel R, Wong BY, et al. The ABL genes in normal and abnormal cell development. Crit Rev Oncog 1996;7:33-48.

9 O’Neill AJ, Cotter TG, Russell JM, et al. Abl expression in human fetal and adult tissues, tumours and tumour microvessels. F Pathol 1997;183:325-9.

10 Bjornsson J, McLeod RA, Unni KK, et al. Primary chondrosarcoma of the long bones and limb girdles. Cancer 1998;83:2105-19.

11 Staunton MJ, Gaffney EF. Tumor type is a determinant of susceptibility to apoptosis. Am F Clin Pathol 1995;103:300-7.

12 Farnum CE, Wilsman NJ. Morphologic stages of the terminal hypertrophic chondrocyte of growth plate cartilage. Anat Rec 1987;219:221-32.

13 Farnum CE, Turgai J, Wilsman NJ. Visualization of living terminal hypertrophic chondrocytes of growth plate cartilage in situ by differential interference contrast microscopy and time-lapse cinematography. $\mathcal{f}$ Orthop Res 1990;8:750-63.
14 Gibson G, Lin D-L, Roque M. Apoptosis of terminally differentiated chondrocytes in culture. Exp Cell Res 1997;233: ferentiate

15 Zenmyo M, Komiya S, Kawabata R, et al. Morphological and biochemical evidence for apoptosis in the terminal hypertrophic chondrocytes of the growth plate. 7 Pathol 1996;180:430-3.

16 Gibson GJ, Kohler WJ, Schaffler MB. Chondrocyte apoptosis in endochondral ossification of chick sterna. Dev Dyn 1995;203:468-76.

17 Enomoto-Iwamoto M, Iwamoto M, Mukudai Y, et al. Bone morphogenetic protein signaling is required for maintenance of differentiated phenotype, control of proliferation, and hypertrophy in chondrocytes. F Cell Biol 1998;140: $409-18$.

18 Roach HI, Erenpresia J, Aigner T. Osteogenic differentiation of hypertrophic chondrocytes involves asymmetric cell of hypertrophic chondrocytes involves asymmetric
divisions and apoptosis. F Cell Biol 1995;131:483-94.

19 Kameda T, Watanabe, Iba H. C-Jun and JunD suppress maturation of chondrocytes. Cell Growth Differ 1997;8: 495-503.

20 Yang C, Li S-W, Helminen HJ, et al. Apoptosis of chondrocytes in transgenic mice lacking collagen II. Exp Cell Res 1997;235:370-3.

21 Kennedy MM, Lucas SB, Russell-Jones R, et al. HHV8 and female Kaposi's sarcoma. F Pathol 1997;183:447-52.

22 Staunton MJ, Gaffney EF. Apoptosis: basic concepts and potential significance in human cancer. Arch Pathol Lab Med 1998;122:310-19.

23 Leoncini L, Del Vecchio MT, Megha T, et al. Correlations between apoptotic and proliferative indices in malignant non-Hodgkin's lymphomas. Am f Pathol 1993;142:755-63.

24 Mc Menamin ME, O Neill AJ, Gaffney EF. The extent of apoptosis in ovarian serous carcinoma in relationship to mitotic and proliferative indices, p53 expression and survival. F Clin Pathol: Mol Pathol 1997;50:242-6.

25 O Neill AJ, Staunton MJ, Gaffney EF. Apoptosis occurs independently of $\mathrm{bcl}-2$ and $\mathrm{p} 53$ over-expression in non-small cell lung carcinoma. Histopathology 1996;29:4550.

26 Lipponen PK, Aaltomaa S. Apoptosis in bladder cancer as related to standard prognostic factors and prognosis. $\mathcal{F}$ Pathol 1994;173:333-9.

27 Evan G, Littlewood T. A matter of life and cell death. Science 1998;281:1317-22.

28 Hasegawa T, Seki K, Yang P, et al. Differentiation and proliferative activity in benign and malignant cartilaginous tumours of bone. Hum Pathol 1995;26:838-45.

\section{Fournal of Clinical Pathology - http://www.molpath.com}

Visitors to the world wide web can now access the fournal of Clinical Pathology either through the BMJ Publishing Group's home page (http://www.bmjpg.com) or directly by using its individual URL (http://www.molpath.com). There they will find the following:

- Current contents list for the journal

- Contents lists of previous issues

- Members of the editorial board

- Information for subscribers

- Instructions for authors

- Details of reprint services.

A hotlink gives access to:

- BMJ Publishing Group home page

- British Medical Association web site

- Online books catalogue

- BMJ Publishing Group books.

The web site is at a preliminary stage and there are plans to develop it into a more sophisticated site. Suggestions from visitors about features they would like to see are welcomed. They can be left via the opening page of the BMJ Publishing Group site or, alternatively, via the journal page, through "about this site". 